\title{
Determinação de HPAs em preparações comerciais com base em pimenta
}

Este estudo avalia a presença e os níveis de hidrocarbonetos policíclicos aromáticos (HPAs) em amostras comercias de pimentas, usando extração sob ultrassom acompanhada de etapas de cleanup e determinação por cromatografia gasosa acoplada à espectrometria de massas. Foram avaliados os 16 HPAs listados como contaminantes ambientais prioritários pela United States Environmental Protection Agency e utilizados como parâmetros a soma de Benzo(a)Antraceno, Criseno, Benzo(b)Fluoranteno e Benzo(a)Pireno os HPAs 4, indicadores usados pela Agência Europeia de Segurança Alimentar para a presença desta classe de hidrocarbonetos em alimentos e a soma total para HPAs de maior peso molecular (Benzo(k)Fluoranteno, Benzo(b)Fluoranteno, Benzo(g, h, i)Perileno, Indeno(1, 2, 3-cd)Pireno estabelecida pela German Society for Fat Science. Foram encontrados os dezesseis HPAs, destacando-se como majoritários o Fenantreno, seguido pelo Criseno, Benzo(a)Antraceno, Acenaftileno e por último o Fluoreno. As concentrações de HPAs 4 variaram de $92,2 \mu \mathrm{Kg}-1 \pm 1,9 \%$ a $1858,3 \mu \mathrm{g} \mathrm{Kg}-1 \pm 14,9 \% \mathrm{nas}$ amostras de pimentas. A soma dos HPAs prioritários nas amostras comerciais de pimenta variou de $2958,7 \mu \mathrm{g} \mathrm{Kg}-1 \pm 4,5 \%$ e $34640,1 \mu \mathrm{g} \mathrm{Kg}-1 \pm 17,5 \%$. As concentrações de HPAs nas amostras de pimentas ultrapassaram os valores permitidos pelo órgão de controle em alimentos, resultando em níveis que podem causar danos à saúde.

Palavras-chave: Contaminantes ambientais; Especiarias; Segurança Alimentar; GC/MS.

\section{Determination of PAHs in commercial preparations based on peppers}

This study assesses the presence and levels of polycyclic aromatic hydrocarbons (PAHs) in commercial pepper samples, using extraction under ultrasound followed by steps of cleanup and determination by gas chromatography coupled to mass spectrometry. The 16 PAHs listed as priority contaminants by the United States Environmental Protection Agency were evaluated and the sum of the four PAHs (Benzo(a)Anthracene, Chrysene, Benzo(b)Fluoranthene, Benzo(a)Pyrene) indicated by the European Food Safety Agency for the presence of PAHs in food and the total sum for higher molecular weight PAHs (Benzo(k)Fluoranthene, Benzo(b)Fluoranthene, Benzo(g,h, i)Perylene, Indene(1, 2, 3-cd)Pyrene established by the German Society for Fat Science were used as parameters in the samples. The sixteen PAHs listed in most of the samples were found, standing out as major Phenanthrene, followed by Chrysene, Benzo(a)Anthracene, Acenaphthylene, and finally Fluorene. The concentrations of PHAs 4 (EU) ranged from $92.2 \mu \mathrm{g} \mathrm{kg}-1 \pm 1.9 \%$ to $1858.3 \mu \mathrm{g} \mathrm{kg}-1 \pm 14.9 \%$ in the pepper samples. The sum of the priority PAHs in the commercial pepper samples ranged from $2958.7 \mu \mathrm{g} \mathrm{kg}-1 \pm 4.5 \%$ to $34640.1 \mu \mathrm{g} \mathrm{kg}-1 \pm 17.5 \%$. The concentrations of PAHs in the pepper samples exceeded the values allowed by the control organ in food, resulting in levels that can cause damage to health.

Keywords: Environmental Contaminants; Spices; Food Security; GC/MS

Topic: Desenvolvimento, Sustentabilidade e Meio Ambiente

Reviewed anonymously in the process of blind peer
Received: 06/06/2021

Approved: 28/06/2021
Monise Ellen Alves da Silva

Instituto Federal Sul-Rio-Grandense, Brasil http://lattes.cnpq.br/2095998888667649

monise.ellen.alves@gmail.com

Daniel Ricardo Arsand (D)

Instituto Federal Sul-Rio-Grandense, Brasil http://lattes.cnpq.br/2243464346389170 http://orcid.org/0000-0002-6167-491X

daniel.arsand@gmail.com

\section{Charles Soares Huber (iti}

Instituto Federal Sul-Rio-Grandense, Brasil http://lattes.cnpq.br/9439672740058859 http://orcid.org/0000-0003-0205-2261 chahuber@gmail.com
Pedro José Sanches Filho (iD

Instituto Federal Sul-Rio-Grandense, Brasi

http://lattes.cnpq.br/9785390634457316

http://orcid.org/0000-0002-9852-8940

pjsans@ibest.com.br
Referencing this:

SILVA, M. E. A.; ARSAND, D. R.; HUBER, C. S.; SANCHES FILHO, P. J.. Determinação de HPAs em preparações comerciais com base em pimenta. Revista Ibero Americana de Ciências Ambientais, v.12, n.6, p.483-498, 2021. DOI: http://doi.org/10.6008/CBPC21796858.2021 .006 .0040 


\section{INTRODUÇÃO}

Os hidrocarbonetos estão listados como alguns dos principais contaminantes ambientais, pois são gerados facilmente a partir de fontes naturais e por diferentes atividades antrópicas, apresentando grande dispersão ambiental (HARTZELL et al., 2018). Dentre eles, os hidrocarbonetos policíclicos aromáticos (HPAs) apresentam alta toxicidade à biota devido seu potencial carcinogênico e mutagênico (GARCIA et al., 2014).

Os HPAs são moléculas diversificadas com mais de cem compostos orgânicos, constituídos apenas de carbono e hidrogênio, formados por dois ou mais anéis aromáticos condensados, cada qual contendo cinco ou seis átomos de carbonos (BUDZINKI et al., 2004). A grande parte da formação destes compostos se dá pela combustão incompleta de compostos orgânicos e estão presentes na queima incompleta de óleos, petróleo e seus derivados, nos escapamentos de veículos, na fumaça de cigarros e assemelhados, na calefação, na queima de carvão ou madeira, na incineração de rejeitos, entre outros (PEREIRA NETTO et al., 2000; HARTZELL et al., 2018). Após formação, os HPAs, distribuem-se nos compartimentos ambientais em proporções que dependem de suas propriedades físico-químicas e das características de cada compartimento ambiental (CHEN et al., 2017).

De acordo com Pietzch (2017), esses compostos podem se dividir em duas classes: compostos com baixa massa molecular (Naftaleno, Acenaftileno, Acenafteno, Fluoreno, Fenantreno e Antraceno) e com alta massa molecular (Fluoranteno, Pireno, Benzo(a)Antraceno, Criseno, Benzo(k)Fluoranteno, Benzo(b)Fluoranteno, Benzo(a)Pireno, Dibenzo(a,h)Antraceno, Benzo(g,h,i)Perileno, Indeno(1,2,3cd)Pireno). Nestes hidrocarbonetos estão os 16 HPAs listados pela States Environmental Protection Agency (USEPA) como contaminantes prioritários e suas estruturas moleculares estão apresentadas na Figura 1 (SUN et al., 2018; TU et al., 2018).
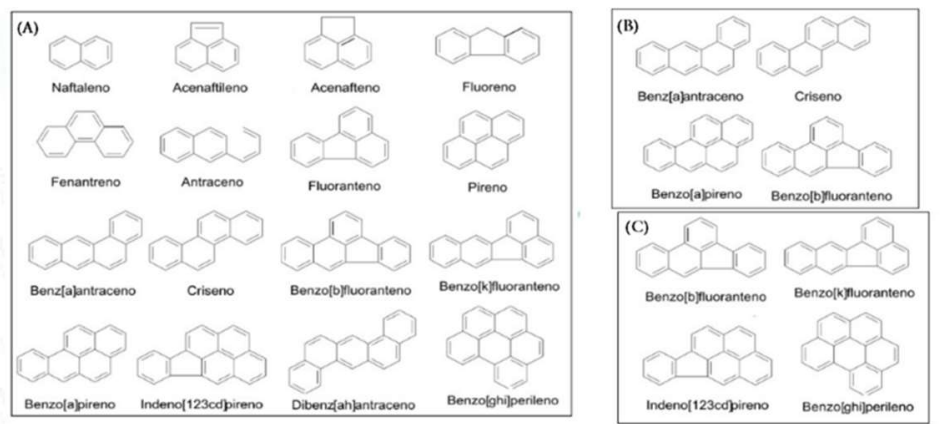

Figura 1: (A) Estruturas moleculares dos 16 HPAs contidos na lista de contaminantes prioritários pela USEPA; (B) HPA4 - European Food Safety Authority; (C) HPAs de alta massa molecular pela German Society for Fat Science. Fonte: Pietzsch (2017) e Bettin et al. (2005).

Embora dezenas de HPAs tenham sido isolados e estudados, destacam-se os avaliados em alimentos e reconhecidos por organizações internacionais como World Health Organization (WHO), Food and Agriculture Organization (FAO) e International Agency for Research on Cancer (IARC) pelo potencial carcinogênico. Os 13 desses compostos em evidência, são: o Benzo(a)Antraceno (B(a)A), o Criseno (ChR), o Benzo(b)Fluoranteno (B(b)F), o Benzo(j)Fluoranteno (B(j)F), o Benzo(k)Fluoranteno (B(k)F), o Benzo(a)Pireno (B(a)P), o Dibenzo(a,h)Antraceno (DhA), o Dibenzo(a,e)Pireno (DeP), o Dibenzo(a,h)Pireno (DhP), o 
Dibenzo(a,i)Pireno (DiP), o Indeno(1,2,3-dc)Pireno (IcP) e o 5-Metilcriseno (5MC) (FAO, 2008). Dentre estes compostos, o Benzo(a)Pireno (BaP) apresenta como efeito mais significativo comprovado a carcinogenicidade (IARC, 2012).

Assim, a exposição ocupacional a misturas contendo HPAs está associada a diversos tipos de câncer como: os de pulmão, pele, bexiga, entre outros (INCA, 2018). Sendo uma classe com centenas de compostos e imprescindível seu monitoramento, a Contaminants in the Food Chain (CONTAM) juntamente com a European Food Safety Authority (EFSA) atribuíram como indicadores dos níveis de HPAs em alimentos o Criseno, Benzo(a)Antraceno, Benzo(b)Fluoranteno e o Benzo(a)Pireno, sendo esses compostos definidos como os HPAs 4 (EFSA, 2008). Na Alemanha, a German Society for Fat Science também fixou um limite total de HPAs em alimentos para o teor de HPAs de alta massa molecular (BETTIN et al., 2005).

Os alimentos e bebidas são uma das maiores fontes de exposição humana aos HPAs (LIN et al., 2005; ROZENTALE et al., 2017; BERTINETTI et al., 2018; SILVA et al., 2020). A ocorrência de contaminação em alimentos por HPAs provém de diferentes formas, incluindo o contato com a água, ar e/ou solo poluídos e pelo processamento de alimentos, principalmente os que apresentam elevadas temperaturas (secagem, defumação, tostagem, grelhagem) (CHUNG et al., 2011; JAHURUL et al., 2013). A presença e as concentrações destes compostos estão relacionadas também com suas características físico-químicas como elevada lipofilicidade, a mesma propriedade que determina sua absorção, distribuição e bioacumulação no organismo humano (CARUSO et al., 2008; PURCARO et al., 2012; KUHN et al., 2020).

As especiarias, incluindo os diversos tipos de pimentas, vêm sendo usadas para atribuir sabor, cor, aroma e preservação para alimentos e bebidas. Elas servem de ingredientes para alimentos processados, produtos à base de carne, laticínios e produtos de panificação e maioria dos produtos culinários (EFSA, 2008; EMBRUSCADO, 2015; SCHAARSCHMIDT, 2016; ROZENTALE et al., 2017).

A qualidade organoléptica e comercial dessas especiarias pode ser afetada pela origem, processos envolvidos na produção, presença de corantes e outros aditivos alimentares, sendo muitos desses proibidos. Os principais grupos químicos presentes em especiarias e que apresentam riscos à saúde, compreendem micotoxinas, resíduo de pesticidas, metais pesados, contaminantes orgânicos persistentes que incluem os HPAs (MORET et al., 2010; MARTENA et al., 2011; REINHOLDS et al., 2016; SCHAARSMIDT, 2016).

De acordo com Empresa Brasileira de Pesquisa Agropecuária (EMBRAPA), não existe uma estimativa exata da produção de pimenta no país, mas acredita-se que são aproximadamente 5 mil hectares de área cultivada por ano, gerando uma produção de 75 mil toneladas de especiarias. A grande parte da produção é cultivada por pequenos agricultores de diversas regiões brasileiras. Na região Sudeste consome-se principalmente a pimenta doce do tipo americana, pimenta cambuci, malagueta e cumari vermelha. $\mathrm{Na}$ região Nordeste, predominam as pimentas malagueta e pimenta de cheiro. Na região Norte, as pimentas mais apreciadas são a murupi, cumari do Pará e a pimenta de cheiro, e na região Centro-Oeste, tradicionalmente são cultivadas e consumidas pimentas bode, malagueta, cumari do Pará, dedo de moça e mais recentemente pimenta de cheiro, anteriormente importada do Pará e atualmente já cultivada em Goiás. Existem poucos resultados sobre estudos de contaminação de HPAs em pimentas. Pela base de dados 
Scopus $^{1}$, somente 13 publicações foram encontradas nos últimos 20 anos abordando o assunto.

Considerando o grau de toxicidade desses compostos e a necessidade de avaliar o seu aporte à dieta local por meio do consumo de pimentas, o objetivo deste estudo foi determinar a presença e níveis de HPAs em amostras comerciais de pimenta por Cromatografia gasosa acoplada à espectrometria de massas (do inglês gas chromatography coupled to mass spectrometry - GC/MS).

\section{MATERIAIS E MÉTODOS}

Para o desenvolvimento deste estudo foram selecionadas cinco amostras de pimentas: AP1- Pimenta Merken (Capsicum van.lungun); AP2-Páprica Picante Defumada (Capsicum annuum); AP3-Pimenta Síria; AP4Pimenta Calabresa (Capsicum baccatum) e AP5-Pimenta Caiena em Pó (Capsicum frutescens), adquiridas em estabelecimentos comerciais locais aleatórios, no período de novembro de 2018 à janeiro de 2019. Após a aquisição, as amostras foram armazenadas em recipientes de vidro (transparente) previamente descontaminados e colocados no dessecador.

A descontaminação feita com solução Extran (MA02 - Neutro, $\mathrm{MERCK}^{\circledR}$ ) e água corrente (torneira), lavados com água destilada e posteriormente com água deionizada; após os recipientes foram levados à estufa a $105^{\circ} \mathrm{C}$ por 2 horas e, por fim, lavados com $1 \mathrm{~mL}(1: 1 \mathrm{v} / \mathrm{v})$ da solução de acetona e hexano e secos em estufa a $105^{\circ} \mathrm{C}$ por 30 minutos (ABNT, 2015). As determinações de umidade e concentração de HPAs nas amostras foram realizadas em triplicata.

\section{Solventes e reagentes}

A determinação dos HPAs se deu por meio do uso de duas misturas padrão (MP1 e MP2). A MP1 consiste em 16 HPAs (1-Naf, 2-Acl, 3-Ace, 4-Fl, 5-Fen, 6-An, 7-Flu, 8-Pir, 9-B(a)A, 10-ChR, 11-B(b)F, 12-B(b)F, 13-B(b)P, 14-IP, 15-DhA, 16-B(ghi)P, a $2000 \mathrm{mg} \mathrm{L}^{-1}$ adquirido da Supelco (BELLEFONTE, PA, EUA). A segunda mistura (MP2) refere-se aos HPAs deuterados (Fenantreno-D10, Chrysene-D12, Perileno-D12), a $2000 \mathrm{mg} \mathrm{L}^{-1}$ adquirido da Supelco (Sulpeco park, Bellefonte, PA, EUA). Os solventes e reagentes usados para extração e cleanup por cromatografia líquida preparativa em coluna com: hexano, diclorometano, metanol, acetona, sulfato de sódio anidro, neutral alumina (type 507, 70-290 mesh ASTM), e sílica gel (70-230 mesh ASTM) adquiridos da Merck Chemical Industries. Toda a água ultrapura utilizada no estudo foi produzida com auxílio de um sistema de purificação de água Milli-Q (Millipore Co.)

\section{Determinação de umidade}

Como caracterização preliminar, foi determinada a umidade segundo o método indireto onde $2.0 \mathrm{~g}$ de cada amostra foram mantidas em estufa a $100-105^{\circ} \mathrm{C}$ por 24 horas, para desidratação. A umidade foi calculada por diferença de peso e expressa em porcentagem (AOAC, 2002), conforme Equação 1 onde $m_{i}$ corresponde à massa inicial e $m_{\mathrm{f}}$ à massa final.

\footnotetext{
${ }^{1}$ www.scopus.com
} 


$$
\operatorname{Umidade}(\%)=\frac{(m i-m f)}{m i} \cdot 100
$$

\section{Extração de HPAs em preparações comerciais com base em pimenta}

A extração de HPAs das amostras foi fundamentada na técnica de Lin et al. (2005), utilizada para análises em chás. As amostras ( $\pm 2,0 \mathrm{~g}$ ) foram adicionadas a $30,0 \mathrm{~mL}$ de solução de hexano/acetona (94:6, $\mathrm{v} / \mathrm{v}$ ) e sonificadas (frequência de $40 \mathrm{kHz}$ ) em um banho termostatizado a $30^{\circ} \mathrm{C}$ (USC 4800A Unique) por 15 minutos. O extrato foi levado à filtração simples e a fase líquida levada à rota-evaporação para remoção dos solventes.

Para avaliar o desempenho da extração, uma massa de 2,0 g (amostra AP1) foi fortificada com $20 \mu \mathrm{g}$ $\mathrm{kg}^{-1}$ da mistura MP2 (Fenantreno - D10, Chrysene - D12, Perylene - D12).

\section{Cromatografia Líquida Preparativa e coluna (CLP) - métodos de Cleanup}

Para a pré-concentração e cleanup, dois métodos foram estudados. No método 1, os extratos foram fracionados em coluna de vidro ( $21 \times 1,1 \mathrm{~cm}$ d.i.), empacotada com 1,0 g de $\mathrm{Na}_{2} \mathrm{SO}_{4}$ anidro ativado, 3,2 $\mathrm{g}$ de sílica gel 60 (70-230 mesh ASTM) 5\% desativada, 1,8 g de alúmina desativada a 2\% e 1,0 g de $\mathrm{Na}_{2} \mathrm{SO}_{4}$ anidro. O extrato foi aplicado na coluna e eluído com $20,0 \mathrm{~mL}$ de hexano para a fração $\mathrm{F} 1$ (alifático), 20,0 mL de uma mistura de diclorometano/hexano $(1: 5, \mathrm{v} / \mathrm{v})$ seguidos de $20,0 \mathrm{~mL}$ de uma mistura de diclorometano/hexano (1:1, v/v) que gerou a fração F2 (HPAs) (UNEP, 1992).

Para o método 2, os extratos foram fracionados em coluna de vidro $(21$ x 1,1 cm d.i.) preenchida com 3,0 g de sílica gel 60 (malha 70-230 ASTM), desativada com 15\% de água e 2,0 g de $\mathrm{Na}_{2} \mathrm{SO}_{4}$ anidro. $\mathrm{O}$ extrato foi aplicado na coluna e eluído com $5,0 \mathrm{~mL}$ de hexano para a fração $\mathrm{F} 1$ (alifático) e 25,0 $\mathrm{mL}$ de diclorometano/hexano (1:4, v/v) para a fração F2 (HPAs) (BETTIN et al., 2005; SANCHES FILHO et al., 2021).

Para o desenvolvimento desta etapa, foram utilizados os extratos de Pimenta Merken (Capsicum van.Lungum) e foram submetidos aos dois procedimentos de cleanup.

\section{Análise Cromatográfica GC/MS}

Os volumes das frações F1 e F2 foram recompostos para 1,0 mL com diclorometano, sob uma suave corrente de nitrogênio gasoso de pureza 99,90\% e submetidos à determinação por cromatografia gasosa acoplada à espectrômetro de massas.

A alíquota de $1 \mu \mathrm{L}$ de cada extrato (em triplicata) foi injetada automaticamente em modo splitless em cromatógrafo gasoso acoplado ao espectrômetro de massas (GC/MS - QP2010 Ultra Shimadzu), sendo equipado com auto amostrador AOC-20i Shimadzu. As condições cromatográficas são mostradas na Tabela 1. A quantificação dos HPAs foi realizada em modo SIM (single ion monitoring). A identificação dos compostos foi baseada no tempo de retenção de cada analito em comparação com os da mistura padrão (MP1) e m/z correspondentes a cada composto. 
Tabela 1: Condições cromatográficas para a determinação de HPAs.

\begin{tabular}{|c|c|}
\hline Parâmetro & Especificação \\
\hline Coluna & $\begin{array}{l}\text { OV-5ms coluna ( } 30 \mathrm{~m} \times 0,25 \mathrm{~mm} \text { d.i. } \times 0,25 \mu \mathrm{m} \text { espessura de filme (J \& W Scientific, Folson, CA, } \\
\text { USA) }\end{array}$ \\
\hline Modo de Injeção & Splitless \\
\hline Temperatura do injetor & $280^{\circ} \mathrm{C}$ \\
\hline Temperatura da interface & $200^{\circ} \mathrm{C}$ \\
\hline lonização & Elétron-Impacto (EI) em $70 \mathrm{Ev}$ \\
\hline Gás de transporte/fluxo & Hélio $(99,999 \%)(1,0 \mathrm{~mL} \mathrm{~min}-1)$ \\
\hline $\begin{array}{l}\text { Temperatura do forno }(\mathrm{F} 2) \\
\text { Íons Monitorados (modo }\end{array}$ & $\begin{array}{l}\text { Temperatura inicial } 60^{\circ} \mathrm{C}(5 \mathrm{~min}), 8^{\circ} \mathrm{C} / \mathrm{min}, 200^{\circ} \mathrm{C}, 5^{\circ} \mathrm{C} / \mathrm{min}, 300^{\circ} \mathrm{C}(20 \mathrm{~min}) \text {. } \\
\mathrm{m} / \mathrm{z}: 128,152,154,166,178,202,228,252,276,278\end{array}$ \\
\hline SIM) & \\
\hline $\begin{array}{l}\text { HPAs } \\
\text { Íons Monitorados (modo } \\
\text { SIM) } \\
\text { HPAs Deuterados }\end{array}$ & $\mathrm{m} / \mathrm{z}: 188,240,264$ \\
\hline
\end{tabular}

\section{Otimização do método cromatográfico}

Para otimização do método cromatográfico, foi utilizado padrão de $5000 \mu \mathrm{g} \mathrm{L} \mathrm{L}^{-1}$, contendo os $16 \mathrm{HPAs}$ estudados, onde foi cromatografado em modo SCAN para determinação da ordem de saída através dos tempos de retenção e identificação dos principais íons no espectro de massas.

Para avaliação do método cromatográfico, foram construídas curvas de calibração preparadas a partir de padrões que compreendem 16 HPAs diluídos com diclorometano. Os parâmetros para validação analisados foram linearidade, precisão, exatidão, limite de detecção (LD) e limite de quantificação (LQ).

A linearidade foi avaliada por meio da construção de curva de calibração em faixas entre 25 a 5000 $\mu \mathrm{L} \mathrm{L}^{-1}$ dos padrões, onde usou-se pelo menos seis pontos, construída pelo método dos mínimos quadrados e expresso como coeficiente de correlação linear. Para a determinação de LD e LQ, foram calculados com base na análise do sinal do branco considerando 3 vezes o desvio padrão para LD e 10 vezes o desvio padrão para LQ e dividido pelo coeficiente angular das curvas analíticas referente a cada analito (IUPAC, 1997).

Os estudos de precisão do método cromatográfico foram realizados a partir das medidas obtidas para o padrão de $50 \mu \mathrm{g} \mathrm{L}^{-1}(\mathrm{n}=7)$, sendo avaliada por meio do desvio padrão relativo expresso \% (ARDUIM et al., 2019). A exatidão foi avaliada por meio do erro relativo, sendo este a diferença entre o valor encontrado a partir das curvas analíticas para o padrão de $500 \mu \mathrm{g} \mathrm{L}^{-1}$.

\section{Controle de qualidade do método de extração e cleanup selecionados}

No controle de qualidade, foi avaliada a precisão por meio do desvio padrão relativo de cada triplicata em relação aos 16 HPAs analisado nas amostras, e a exatidão e o efeito de matriz, através dos ensaios de recuperação onde uma massa de 2,0 g (AP1) foi fortificada com $20 \mu \mathrm{g} \mathrm{kg}^{-1}$ da mistura MP2 (Fenantreno - D10, Chrysene - D12, Perylene - D12). As recuperações foram calculadas através da razão entre a quantidade determinada de HPAs deuterados e a quantidade de HPAs adicionada, multiplicada por 100.

Os procedimentos realizados foram seguidos com análise do branco para avaliar a potencial interferência de contaminantes no ambiente de trabalho, limpeza da vidraria, bem como, oriundos dos reagentes utilizados. 


\section{Aplicação a amostras de pimenta}

Após a otimização de todas as etapas, a metodologia foi aplicada nas amostras de pimentas.

A avaliação do nível de contaminação das amostras de pimenta foi realizada através da concentração total de HPAs, dos HPAs 4 e da concentração total dos HPAs de alta massa molecular, como são especificados no Regulamento da Comissão das Comunidades Europeias n 835/2011 (EU, 2011) e pela German Society for Fat Science.

\section{RESULTADOS E DISCUSSÃO}

\section{Otimização do método GC/MS}

Com base no estudo da mistura dos 16 HPAs em modo SCAN, foi possível determinar a ordem de saída de cada composto. A Figura 2 apresenta o cromatograma para a mistura padrão contendo os HPAs prioritários, onde foram avaliados os tempos de retenção e seus respectivos espectros de massa. Nos detalhes da imagem do cromatograma (regiões indicadas como A, B e C), observa-se a identificação dos HPAs e a seguir a comparação entre o espectro de massas gerados a partir do padrão Naftaleno e o espectro da biblioteca espectral NIST - 05 do equipamento.

Através dos espectros de massas, verifica-se que o íon molecular corresponde ao íon base para os 16 HPAs. Assim, foram selecionados os íons com razão massa/carga (m/z) 128, 154, 166, 166, 178, 202, 228, 252, 276 e 278 para os HPAs, sendo estes utilizados para a quantificação dos compostos por GC/MS em modo SIM.
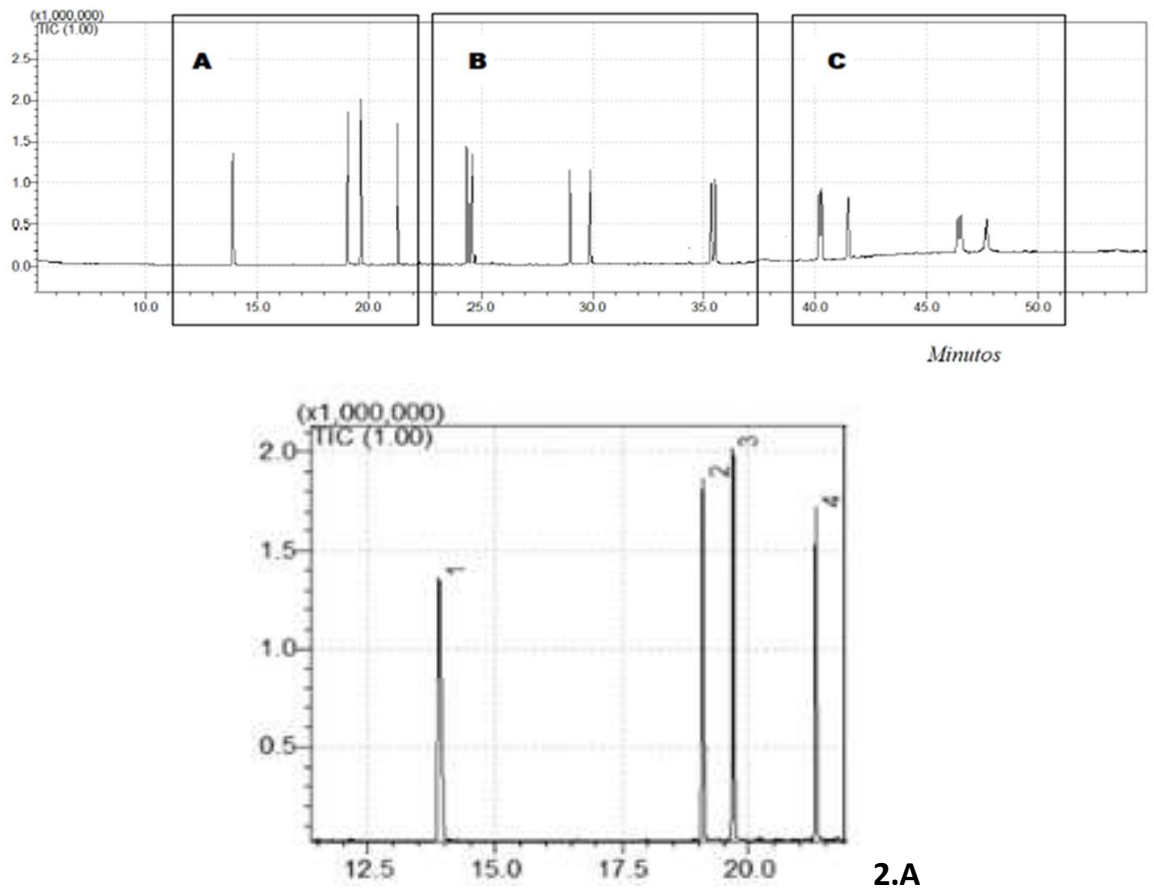

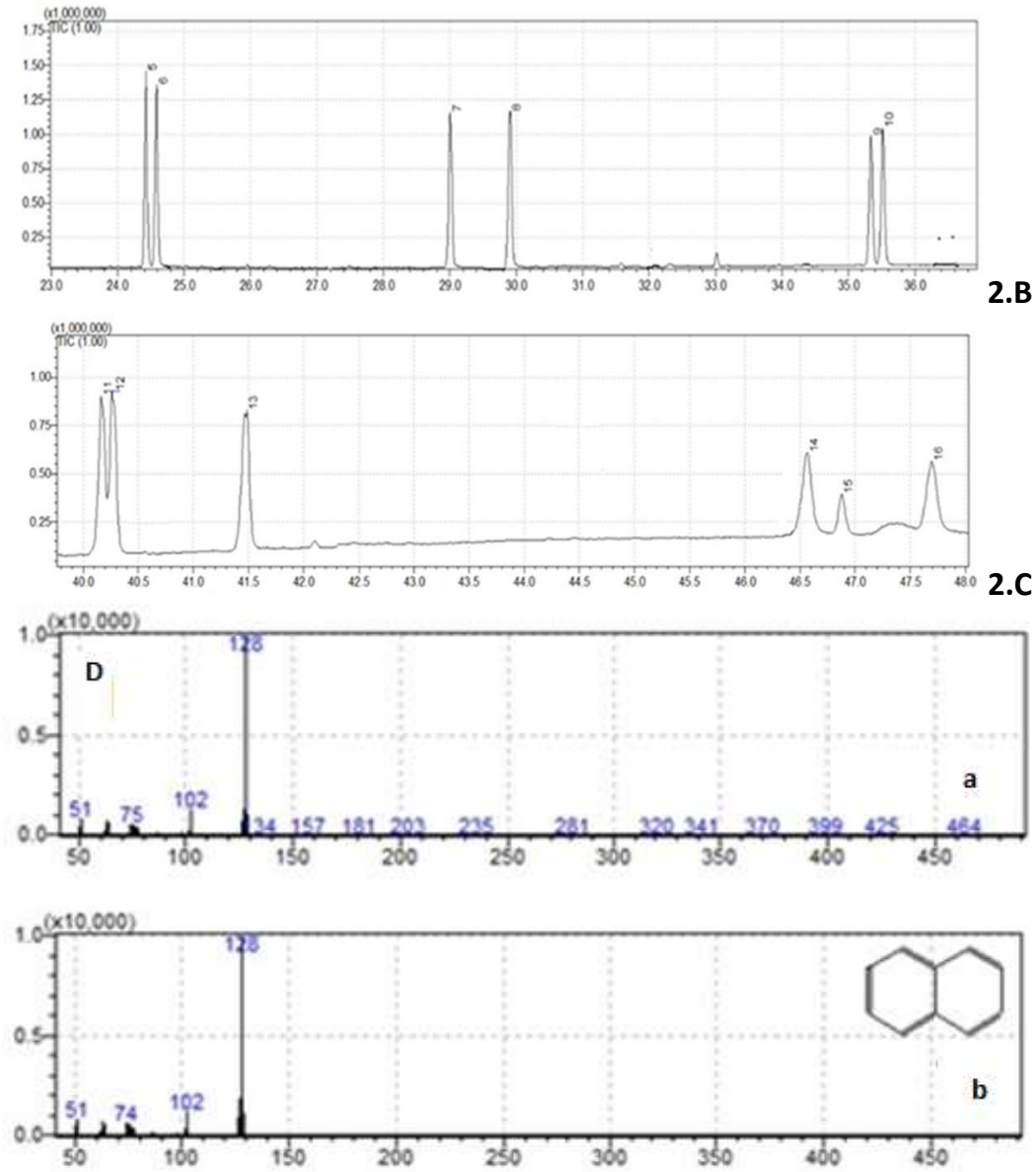

Figura 2. Cromatograma em modo SCAN, com os 16 HPAs prioritários e detalhes com os íons selecionado para o modo SIM ;(A) Identificação do picos: 1 (íon 128) Naftaleno ;2 (íon 152) Acenaftileno; 3 (íon 154) Acenafteno; 4 (íon 166): Fluoreno; (B) picos: 5 e 6 (íon 178) Fenantreno e antraceno; 7 e 8 (íon 202): Fluoranteno e Pireno; 9 e 10 (íon 228) Benzo(a)Antraceno e Criseno; (C) picos 11, 12 e 13 (íon 252): Benzo(k)Fluoranteno, Benzo(b)Fluoranteno, e Benzo(a)Pireno; 14 e 16 (íon 276) Benzo(g,h,i)Perileno e): Indeno(1,2,3cd)Pireno; e 15 (íon 278) Dibenzo(a,h)Antraceno; (D) Comparação entre os espectros de massa obtido a partir do padrão naftaleno (a) e o da biblioteca do equipamento (b).

Para o aumento da sensibilidade e a deteç̧ão no espectrômetro de massas, foi alterada do modo SCAN para o modo SIM, empregando os íons $\mathrm{m} / \mathrm{z}$ correspondente ao íon molecular e o íon base de cada analito e ainda definindo as janelas de tempo para monitoramento dos íons.

A Figura 3 apresenta o cromatograma em modo SIM para o padrão de $500 \mu \mathrm{L} \mathrm{L} \mathrm{L}^{-1}$, indicando as janelas e íons selecionados: os picos relativos aos íons m/z 128 do Naftaleno, 152 do Acenaftileno, 154 do Acenafteno, 166 do Fluoreno, 178 do Fenantreno e Antraceno, 202 do Fluoranteno e Pireno, 228 do Benzo(a)Antraceno e Criseno, 252 do Benzo(k)Fluoranteno, Benzo(b)Fluoranteno e Benzo(a)Pireno, 276 Benzo(g,h,i)Perileno e Indeno(1,2,3-cd)Pireno e o 278 do Dibenzo(a,h)Antraceno. Os picos encontram-se resolvidos, não interferindo nas determinações quantitativas, uma vez que estes são específicos de cada analito. Adicionalmente, o uso em modo SIM realiza um cleanup eletrônico com o desaparecimento de possíveis inteferentes, quando aplicado na determinação dos HPAS nas amostras de pimenta, pois só registra compostos que apresentarem os íons selecionados. Estas condições reunidas foram utilizadas para as demais etapas do estudo. 

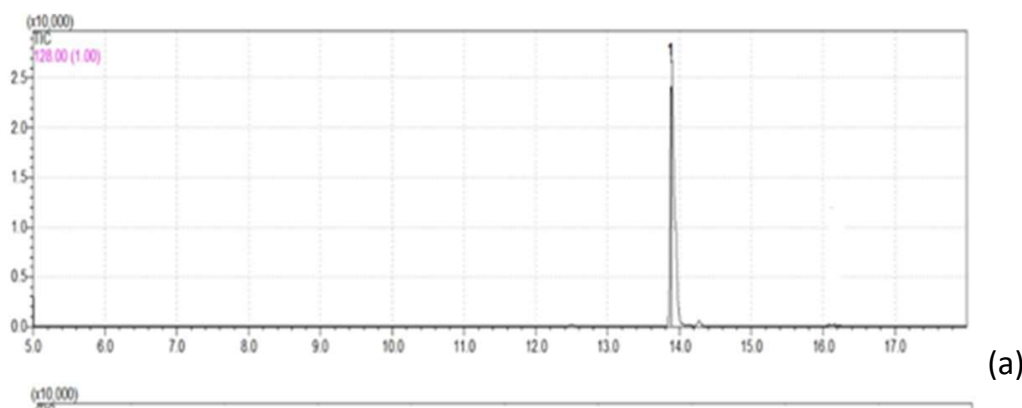

(a)

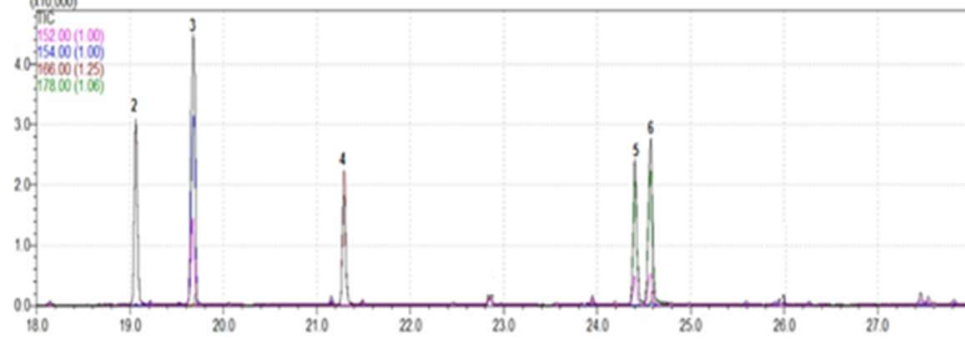

(b)

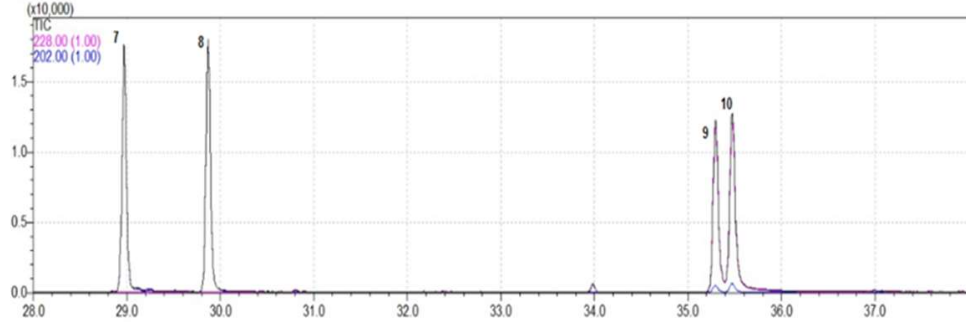

(c)

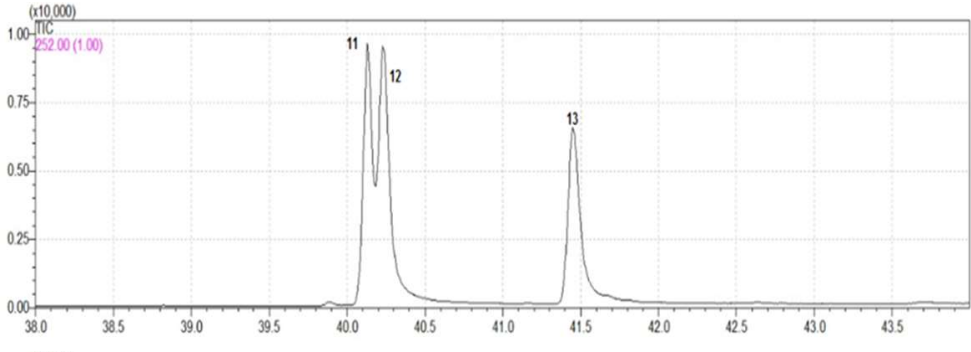

(d)

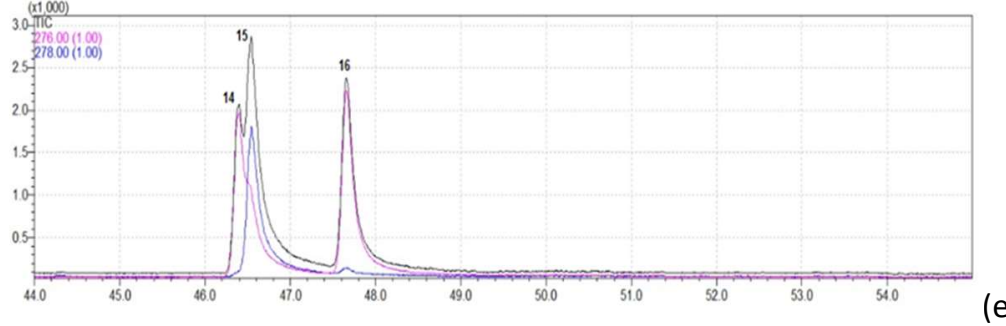

(e)

Figura 3: Cromatogramas apresentando as janelas de tempo de retenção para os 16 HPAs prioritários. (a)Janela - 1 (518 minutos) m/z 128; (b)Janela - 2 (18-28 minutos) m/z 152,154,166,178; (c)Janela - 3 (28-38 minutos) m/z 228,202;

(d)Janela - 4(38-44 minutos) m/z 252; (e)Janela - 5 (44-52 minutos) m/z 276, 278.

\section{Validação do Método Cromatográfico}

A Tabela 2 mostra as figuras de mérito para o método proposto de análise de HPAs em amostras de pimentas. Pode-se observar que para as faixas de trabalho estudadas $\left(25\right.$ a $\left.5000 \mathrm{\mu g} \mathrm{L}^{-1}\right)$ o método apresentou uma resposta linear com coeficientes de correlação $(r)$ variando entre 0,996 a 0,999. Estes valores situam-se acima do preconizado $(>0,9)$ como adequado na validação e métodos quantitativos pelo INMETRO (2003). 0 método apresentou exatidão e precisão para maioria dos analitos, com ER\% -1,1 a 15,3\% e RSDs entre 1,4 a 18,3\% para HPAs. Este valor foi considerado equivalente àquela encontrada por outros autores em análises de HPAs em matrizes vegetais como Rozentale et al. (2017) e Sanches Filho et al. (2021) e adequado para método cromatográficos de acordo com INMETRO (2003). 
Tabela 2: Parâmetros de mérito do método quantitativo Cromatográfico-GC/MS em modo SIM.

\begin{tabular}{|c|c|c|c|c|c|c|c|}
\hline & $Y=a X+b$ & & & $\mu \mathrm{g} \mathrm{Kg}^{-1}$ & $\mu \mathrm{g} \mathrm{L}^{-1}$ & & \\
\hline HPAs & $a$ & $\mathrm{~b}$ & $r$ & $\mathrm{LD} / \mathrm{LQ}$ & $\mathrm{LD} / \mathrm{LQ}$ & RSD\% & ER\% \\
\hline Naf & 320,39 & -39841 & 0,996 & $0,3 / 1,1$ & $0,6 / 1,9$ & 12,0 & $-6,8$ \\
\hline $\mathrm{Acl}$ & 243,86 & -43820 & 0,998 & $0,4 / 1,3$ & $0,7 / 2,3$ & 7,5 & $-7,7$ \\
\hline Ace & 182,11 & 3499 & 0,997 & $0,5 / 1,7$ & $0,9 / 3,0$ & 0,8 & $-0,5$ \\
\hline $\mathrm{Fl}$ & 168,82 & -37619 & 0,998 & $0,5 / 1,8$ & $1,0 / 3,3$ & 4,5 & $-3,6$ \\
\hline Fen & 225,76 & -6200 & 0,996 & $0,4 / 1,4$ & $0,7 / 2,4$ & 13,7 & 1,6 \\
\hline An & 232,11 & -61899 & 0,996 & $0,4 / 1,3$ & $0,7 / 2,4$ & 18,3 & 5,9 \\
\hline Flu & 194,55 & -29855 & 0,998 & $0,4 / 1,4$ & $0,8 / 2,6$ & 5,8 & $-10,2$ \\
\hline Pir & 208,46 & -32148 & 0,998 & $0,4 / 1,4$ & $0,7 / 2,4$ & 5,7 & $-10,2$ \\
\hline$B(a) A$ & 145,62 & -19144 & 0,999 & $0,6 / 1,9$ & $1,0 / 3,5$ & 1,4 & $-8,1$ \\
\hline ChR & 144,57 & -23845 & 0,999 & $0,6 / 2,0$ & $1,1 / 3,5$ & 2,6 & 0,7 \\
\hline$B(k) F$ & 109,92 & -12681 & 0,999 & $0,2 / 0,7$ & $0,4 / 1,3$ & 11,0 & $-4,1$ \\
\hline$B(b) F$ & 111,60 & -11603 & 0,999 & $0,2 / 0,7$ & $0,4 / 1,3$ & 6,6 & $-1,1$ \\
\hline$B(a) P$ & 88,75 & -8066 & 0,999 & $0,3 / 0,9$ & $0,5 / 1,6$ & 10,4 & 2,3 \\
\hline BghiP & 67,41 & -23067 & 0,997 & $0,5 / 1,5$ & $0,8 / 2,7$ & 15,6 & 11,9 \\
\hline DahA & 74,89 & -21620 & 0,999 & $0,4 / 1,4$ & $0,7 / 2,5$ & 16,9 & 11,8 \\
\hline IP & 69,00 & -17669 & 0,998 & $0,4 / 1,5$ & $0,8 / 2,7$ & 14,2 & 15,3 \\
\hline
\end{tabular}

$\mathrm{Y}=\mathrm{aX}+\mathrm{b}$ ( $\mathrm{a}$ - coeficiente angular; $\mathrm{b}$ - coeficiente linear); $\mathrm{r}$ - coeficiente correlação linear; LD - limite detecção; LQ - limite de quantificação.

Os LD e LQ considerando a massa de 2,0 g para as amostras de Pimenta (APs) e considerando a recuperação de $100 \%$ variaram de 0,2 - 0,6 $\mu \mathrm{g} \mathrm{kg}^{-1}$ e 0,7 - 2,0 $\mathrm{\mu g} \mathrm{kg}^{-1}$ respectivamente. Observa-se que os maiores valores referem-se a compostos que possuem menor fator de resposta. Os valores obtidos estão em uma faixa mais alta, que os encontrados por Rozentale et al. (2017) em amostras de especiarias diversas, que apresentou variações de LDs de 0,04 a 0,09 $\mu \mathrm{g} \mathrm{kg}^{-1}$. Para o cálculo dos limites de detecção nas amostras de pimenta, consideramos a massa de aproximadamente (2,0 g), logo, massas maiores implicam em menores limites de detecção e quantificação. O regulamento de n 836/2011 (EU, 2011) da Comissão das Comunidades Europeias estabeleceu um limite de detecção $\left(<0,3 \mu \mathrm{g} \mathrm{kg}^{-1}\right)$ e de quantificação $\left(<0,9 \mu \mathrm{g} \mathrm{kg}^{-1}\right)$ somente para análise de benzo(a)pireno. Verificou-se que nesse estudo os limites de LD e LQ para benzo(a)pireno estão de acordo com o limite exigido.

O coeficiente angular(a) reflete a sensibilidade do método analítico. Para os HPAs, observou-se uma queda na sensibilidade com o aumento do peso molecular. Este comportamento pode estar relacionado à redução do fator resposta (Figura 3d, 3e), que também prejudica a exatidão e precisão dos métodos.

\section{Resultados da comparação dos métodos de Cleanup}

A Tabela 3 apresenta os níveis de HPAs encontrados pelos dois métodos de cleanup para a amostra AP1.

Tabela 3: Concentrações de HPAs em $\mu \mathrm{kg}^{-1}$ e RSD\% em base seca, determinados na comparação dos métodos de cleanup para amostras AP1.

\begin{tabular}{|c|c|c|c|c|}
\hline \multirow{3}{*}{ Compostos } & \multicolumn{2}{|l|}{ Método 1} & \multicolumn{2}{|l|}{ Método 2} \\
\hline & Média & RSD & Média & RSD \\
\hline & $\mu g \mathrm{~kg}^{-1}$ & $\%$ & $\mu g \mathrm{~kg}^{-1}$ & $\%$ \\
\hline Naf & 146,7 & 6,9 & 224,9 & 2,4 \\
\hline $\mathrm{Acl}$ & 308,8 & 5,8 & 1473,7 & 11,8 \\
\hline Ace & 209,6 & 10,7 & 371,3 & 6,0 \\
\hline $\mathrm{FI}$ & 279,7 & 4,8 & 1279,0 & 10,9 \\
\hline Fen & 2608,8 & 10,4 & 13162,6 & 18,0 \\
\hline An & 531,8 & 8,1 & 3157,7 & 15,7 \\
\hline Flu & 801,3 & 4,1 & 6500,7 & 15,9 \\
\hline
\end{tabular}




\begin{tabular}{lllll} 
Pir & 686,5 & 2,0 & 5490,1 & 14,5 \\
BaA & 143,7 & 1,7 & 570,5 & 9,2 \\
ChR & 212,1 & 2,8 & 998,8 & 15,8 \\
BkF & 101,2 & 3,4 & 133,6 & 16,5 \\
BbF & 74,3 & 0,7 & 126,4 & 17,5 \\
BaP & 63,5 & 4,3 & 162,5 & 18,0 \\
BghiP & 196,9 & 3,4 & 204,6 & 9,3 \\
DahA & 402,5 & 9,2 & 403,5 & 9,5 \\
IP & 373,1 & 10,7 & 380,2 & 2,9 \\
\hline
\end{tabular}

Os resultados apresentados na Tabela 3 mostram que o método 2 resulta em níveis mais altos de HPAs que o método 1, principalmente para os compostos mais leves, indicando que o método 2 recupera mais compostos. A perda maior no método 1 pode estar relacionada ao volume maior de solvente na eluição da fração 2 a ser evaporada, o que favorece a perda dos analitos mais leves. Com RSD variando de 1,8 e 12,9\%, o método apresenta precisão aceitável (inferiores a 20\%) para a faixa de concentração de $\mu \mathrm{kg}^{-1}$ (BRITO et al., 2003). Sendo assim, e considerando ser mais econômico e ambientalmente amigável, pois consome menos solvente orgânicos e menos reagentes, se utilizou o método 2 nos estudos de recuperação e para análise das demais amostras de pimentas.

A tabela 4 apresenta os resultados para estudo de recuperação da amostra fortificada com HPAs deuterados.

Tabela 4: O estudo de recuperação para amostras de AP $\left(\mu \mathrm{Kg}^{-1}\right) \pm$ desvio padrão relativo ( $\left.\pm \mathrm{RSD} \%\right)$.

\begin{tabular}{llll}
\hline & AP $\mu \mathrm{g} \mathrm{Kg}^{-1}$ & & \\
\hline & Valor & & \\
HPAs & Fortificado & Valor Encontrado & Recuperação (\%) \\
\hline Phenanthrene D10 & 20,0 & $19,8 \pm 8,4$ & 99,0 \\
Chrysene D12 & 20,0 & $18,9 \pm 8,7$ & 94,5 \\
Perylene D12 & 20,0 & $18,9 \pm 9.0$ & 98,8 \\
\hline
\end{tabular}

Os valores descritos na Tabela 4 são equivalentes aos publicados por outros autores que já trabalharam com matrizes desta natureza. Rozentale et al. (2017) encontraram valores para recuperações na faixa de 72 a 107\%, enquanto Hwang et al. (2019) de 91,4 a 103,1\%. O RSD está de acordo com os dados apresentados na Tabela 3 e confirma a precisão adequada do método.

Além disso, estes valores atendem às exigências do regulamento UE 835/2011 e do parecer da EFSA em conjunto com o CONTAM, que estabelece limites de recuperações de 50 a 120\% (EFSA, 2008).

\section{Resultados em amostra de Pimentas}

Na caracterização preliminar, foi determinada a umidade das cinco amostras. A Tabela 5 mostra o teor de umidade das amostras para as cinco pimentas analisadas.

Tabela 5: Caracterização preliminar de pimenta: umidade.

\begin{tabular}{l}
\hline Amostras Umidade (\% \pm RSD\%) \\
\hline AP1 $11,1 \pm 7,8$ \\
AP2 $10,7 \pm 5,6$ \\
AP3 $8,2 \pm 2,5$ \\
AP4 $9,3 \pm 0,8$ \\
AP5 7,2 $\pm 2,7$ \\
RSD: desvio padrão relativo. \\
Amostras (AP1): pimenta merken; (AP2): páprica picante defumada; (AP3): pimenta síria; (AP4): pimenta calabresa
\end{tabular}


moída; e (AP5): pimenta caiena em pó.

Em comparação com outros estudos, os valores entre 7,2 a 11,1\% são considerados adequados, segundo a American Spice Trade Association (ASTA, 1999) que recomenda teor máximo de umidade de $11 \%$ para as pimentas Capsicum. Valores similares foram encontrados por Zaki et al. (2013) que obtiveram 8,6 a 10,8\% de umidade em amostras de páprica.

A Tabela 6 apresenta o nível de HPAs determinado neste estudo para as APs. Os HPAs foram encontrados em $100 \%$ das amostras analisadas, predominantemente os compostos de menor peso molecular ( 2 a 4 anéis). A Figura 4 apresenta a comparação entre os cromatogramas em modo SCAN e SIM com a identificação do HPAS na amostra AP1. Pode-se observar a redução do número de picos no modo SIM, descrito como clen up eletrônico, aumento do sinal para os HPAS e sua separação de outros compostos presentes na matriz.
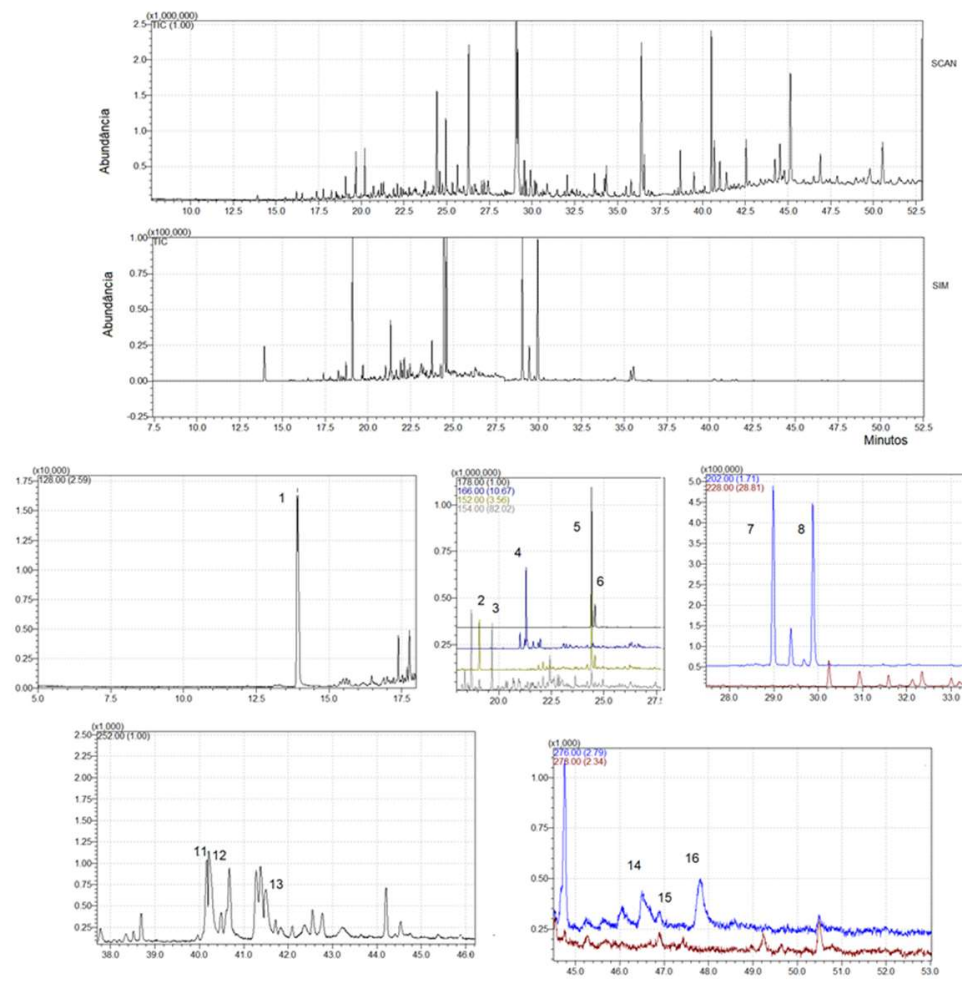

Figura 4: Identificação dos dos HPAS encontrados na amostra AP1 comparação dos cromatogramas em modo SIM e SCAN.

O $\sum$ HPAs totais variaram de $1626,6 \mu \mathrm{g} \mathrm{kg}^{-1} \pm 7,3 \%$ a $34640 \mu \mathrm{g} \mathrm{kg}^{-1} \pm 17,5 \%$. Essas concentrações estão acima das encontradas por Londoño et al. (2015), que apresentaram $\sum$ HPAs entre 509,7 $\mathrm{gg} \mathrm{kg}^{-1}$ a $2746,5 \mu \mathrm{g}$ $\mathrm{kg}^{-1}$, indicando que os níveis de HPAs encontrados neste trabalho são elevados. O principal composto em todas as amostras foi o Fenantreno, seguido pelo Criseno, Benzo(a)Antraceno, Acenaftileno e por último o Fluoreno. A maior presença de HPAs de 3 a 4 anéis é atribuída aos altos teores oriundos de chamas do processo de defumação (AZEVEDO et al., 2013; FASANO et al., 2016).

O Naftaleno, Acenafteno, Acenaftileno, Fluoreno, Fenantreno, Antraceno e Criseno, foram detectados e quantificados em todas as amostras comerciais de pimenta. O Fluoranteno e o Pireno foram detectados apenas nas AP1, AP2 e AP4. Nas demais amostras, estes compostos não foram quantificados em 
função de interferência do éster metílico do ácido linoleico e do éster etílico do ácido 9,12-octadecadienóico, que coeluem com este HPAs apresentando o fragmento $\mathrm{m} / \mathrm{z}$ 202. A situação foi confirmada através das análises das frações em modo SCAN. Somente na AP1 foram detectados todos os 16 HPAs prioritários indicados pela USEPA (1996).

Tabela 6: Concentrações de HPAs em base seca, determinados nas amostras de pimenta.

\begin{tabular}{|c|c|c|c|c|c|c|}
\hline \multirow[t]{2}{*}{ HPAs } & \multicolumn{5}{|c|}{$\mathrm{AP}\left(\mu \mathrm{g} \mathrm{Kg}^{-1} \pm \mathrm{RSD} \%\right)$} & \multirow[t]{2}{*}{ Limites permitidos $\mu \mathrm{g} \mathrm{Kg}^{-1}$} \\
\hline & AP1 & AP2 & AP3 & AP4 & AP5 & \\
\hline Naf & $224,9 \pm 2,4$ & $23,8 \pm 5,9$ & $498,6 \pm 11,3$ & $66,7 \pm 7,7$ & $320,5 \pm 15,1$ & \\
\hline Acl & $1473,7 \pm 11,8$ & $24,9 \pm 13,9$ & $191,4 \pm 16,2$ & $18,4 \pm 6,2$ & $70,7 \pm 7,9$ & \\
\hline Ace & $371,3 \pm 6,0$ & $49,9 \pm 12,9$ & $219,4 \pm 17,7$ & $126,6 \pm 18,2$ & $136,7 \pm 4,3$ & \\
\hline $\mathrm{FI}$ & $1279,0 \pm 10,9$ & $33,3 \pm 7,4$ & $2815,8 \pm 4,8$ & $45,0 \pm 8,2$ & $185,0 \pm 0,7$ & \\
\hline Fen & $13162,6 \pm 18,0$ & $75,5 \pm 17,8$ & $492,2 \pm 7,1$ & $517,6 \pm 18,5$ & $1281,6 \pm 18,9$ & \\
\hline An & $3157,7 \pm 15,7$ & $37,0 \pm 10,9$ & $92,1 \pm 5,5$ & $51,0 \pm 1,1$ & $42,6 \pm 11,3$ & \\
\hline Flu & $6500,7 \pm 15,9$ & $45,2 \pm 2,6$ & Interf & $181,7 \pm 15,7$ & Interf & \\
\hline Pir & $5490,1 \pm 14,5$ & $75,2 \pm 17,6$ & Interf & $994,7 \pm 11,6$ & $405,2 \pm 4,8$ & \\
\hline $\mathrm{BaA}$ & $570,5 \pm 9,2$ & $94,1 \pm 12,7$ & $1949,5 \pm 13,0$ & $\mathrm{Nd}$ & $251,7 \pm 4,8$ & \\
\hline ChR & $998,8 \pm 15,8$ & $64,4 \pm 8,3$ & $501,9 \pm 9,1$ & $21,7 \pm 20,0$ & $116,2 \pm 0,8$ & \\
\hline $\mathrm{BkF}$ & $133,6 \pm 16,5$ & $\mathrm{Nd}$ & $548,0 \pm 9,4$ & $\mathrm{Nd}$ & $156,7 \pm 22,2$ & \\
\hline $\mathrm{BbF}$ & $126,4 \pm 17,5$ & $\mathrm{Nd}$ & $379,1 \pm 7,2$ & $\mathrm{Nd}$ & $197,8 \pm 19,9$ & \\
\hline $\mathrm{BaP}$ & $162,5 \pm 18,0$ & $\mathrm{Nd}$ & $159,0 \pm 18,0$ & $129,6 \pm 5,3$ & $143,1 \pm 16,4$ & $0,03 * * *$ \\
\hline BghiP & $204,6 \pm 9,3$ & $655,5 \pm 17,0$ & $1342,8 \pm 7,9$ & $\mathrm{Nd}$ & $500,9 \pm 2,4$ & \\
\hline DahA & $403,5 \pm 9,5$ & $\mathrm{Nd}$ & $423,2 \pm 26,6$ & $\mathrm{Nd}$ & $\mathrm{Nd}$ & \\
\hline IP & $380,2 \pm 2,9$ & $448 \pm 5,5$ & $659,8 \pm 5,1$ & $\mathrm{Nd}$ & $217,5 \pm 18,2$ & \\
\hline Total & $34640,1 \pm 17,5$ & $1626,9 \pm 7,3$ & $10273,0 \pm 3,6$ & $3976,6 \pm 13,6$ & $2958,7 \pm 4,5$ & \\
\hline $\mathrm{ChR}+\mathrm{BaA}+\mathrm{BbF}+\mathrm{BaP}$ & $1858,3 \pm 14,9$ & $158,3 \pm 5,3$ & $2989,6 \pm 6,5$ & $92,2 \pm 1,9$ & $719,1 \pm 4,1$ & $35.0 * *$ \\
\hline $\mathrm{BbF}+\mathrm{BkF}+\mathrm{BghiP}+\mathrm{IP}$ & $844,8 \pm 9,3$ & $1103,5 \pm 12,1$ & $2197,4 \pm 8,8$ & $\mathrm{Nc}$ & $1504,1 \pm 13,4$ & $5.0^{*}$ \\
\hline
\end{tabular}

*Limite estabelecido para somatório total de HPAs pela German Society for Fat Science; **Regulamento 835/2011/EC; ***European Union (88/388/ec). Interf: interferência. Nd: não detectado. Nc: não calculado. Amostras (AP1): pimenta merken; (AP2): páprica picante defumada; (AP3): pimenta síria; (AP4): pimenta calabresa moída; e (AP5): pimenta caiena em pó.

O nível médio de $\sum$ HPA4 encontrado nas amostras variam de $92,2 \mu \mathrm{g} \mathrm{kg}^{-1} \pm 1,9 \%$ a $1858,3 \mu \mathrm{g} \mathrm{kg}^{-1} \pm$ 14,9\%. Outros estudos, como os realizados por Rozentale et al. (2017), mostraram resultados a partir de 1,01 $\mu \mathrm{g} \mathrm{kg}^{-1}$ a 13,59 $\mu \mathrm{g} \mathrm{kg}^{-1}$. Observou-se, também, que as concentrações encontradas excedem os limites considerados seguros para SHPA4 definidos no regulamento no 835/2011/EC que pré-estabelece o valor de $35 \mu \mathrm{kg}^{-1}$.

A German Society for Fat Science também considera como parâmetro o valor total para HPAs de maior massa molecular para (Benzo(k)Fluoranteno, Benzo(b)Fluoranteno, Benzo(g,h,i)Perileno, Indeno(1,2,3-cd)Pireno) e fixou um limite de $5 \mu \mathrm{g} \mathrm{kg}^{-1}$. O somatório destes compostos só não foi determinado na AP4, variando nas demais amostras de $844,8 \mu \mathrm{g} \mathrm{kg}^{-1}$ a $1504,1 \mu \mathrm{gg}^{-1}$, estando acima do estabelecido pela norma.

Das amostras de pimentas (APs) analisadas, destacam-se as maiores concentrações nas pimentas AP1 e AP3. Esses resultados podem estar relacionados como resultante de seu processo de produção antes de sua comercialização que apresenta um alto nível de defumação, onde a exposição direta às chamas, como no caso da pimenta merken (AP1) que é produzida artesanalmente e envolve exposição direta à fumaça de fogões ou braseiros para desidratação e defumação das pimentas (FASANO et al., 2016; ARCE et al., 2019). Já a pimenta síria (AP3) possui incremento de outras especiarias (pimenta do reino, pimenta-da-Jamaica, canela, cravo e noz-moscada), previamente defumadas, o que leva a maiores índices de HPAs de maior peso 
molecular, explicando as maiores quantidades de HPAs nessa amostra.

As demais pimentas (AP2, AP4 e AP5) analisadas apresentaram níveis mais baixos, mesmo assim, considerando o total destes compostos, os níveis são altos quando comparados com o regulamento de $\mathrm{n}^{\circ}$ 835/2011 (EU, 2011; FASANO et al., 2016; MONAGO-MARAÑA et al., 2016; HWANG et al., 2019).

O BaP detectado nas AP1, AP3, AP4 e AP5 também está acima do estabelecido pela União Europeia (European Union, EU), em uma de suas diretrizes (88/388/EC), que determina um limite para presença deste composto em alimentos defumados que é de $0,03 \mu \mathrm{g} \mathrm{kg}^{-1}$.

Os resultados deste estudo estão bem acima do permitido pelas normas de controle de HPAs em alimentos, estando esses valores altos relacionados ao processo de produção ao qual as pimentas foram expostas.

\section{CONCLUSÕES}

Os resultados permitem concluir que o método de extração por ultrassom acompanhado de préfracionamento em colunas de sílica e análise por GC/MS foi adequado para o estudo e determinação de HPAs presentes em amostras de preparações comerciais com base em pimentas estudadas.

As determinações de HPAs em pimentas mostram níveis elevados atribuídos, principalmente ao seu processo de produção com altos níveis de temperaturas no processo de secagem e defumação. Devido a esses processos, foram quantificados nas amostras principalmente os compostos de menor peso molecular (2 a 4 anéis).

As concentrações de HPAs nas amostras de pimentas estão bem acima do que se é permitida pelas leis que estabelecem limites para HPAs em alimentos, mesmo quando comparados a outras matrizes vegetais e especiarias, explicitando a importância desse e de novos estudos acerca da presença de HPAs em alimentos condimentados.

\section{REFERÊNCIAS}

ABNT. Associação Brasileira de Normas Técnicas. NBR 9.898: Coleta de Amostras. Licenciador Ambiental. ABNT, 2015.

AOAC. Association of Official Analytical Chemists. Official Methods of Analysis of the Association of Analytical Chemists. 17 ed. Gaithersburg: AOAC, 2002.

ASTA. American Spice Trade Association. Cleanliness specifications for spices, seeds, and herbs. Englewoods Cliff: ASTA, 1999.

AZEVEDO, J. A. H.; ARAÚJO, R. S.; SILVA, G. M. M. Hidrocarbonetos Policíclicos Aromáticos Atmosféricos de Fontes Automotivas: uma breve revisão. Holos, v.1, p.102114, 2013. DOI: http://doi.org/10.15628/holos.2013.1234

ARCE, A: MAR, F. C.. Espaços ambíguos e a inovação neoliberal contemporânea: o caso do Merken. Redes Santa Cruz do Sul, 2019.

BERTINETTI, I. A.; FERREIRA, C. D.; MONKS, J. L. F.; FILHO, P. J. S.; CARDOSO, E. M.. Accumulation of polycyclic aromatic hydrocarbons (PAHs) in rice subjected to drying with different fuels plus temperature, industrial processes and cooking. Journal of Food Composition and Analysis, v.6, p.109-15, 2018. DOI: http://doi.org/10.1016/j.jfca.2017.12.009

BETTIN, S. M.; FRANCO, D. W.. Hidrocarbonetos Policíclicos Aromáticos (HPAs) em aguardentes. Food Science and Technology, v.25, p.234-238, 2005. DOI: http://doi.org/10.1590/S0101-20612005000200008

BRITO, N. M.; AMARANTE JUNIOR, O. P.; POLESE, L.; RIBEIRO, M. L.. Validação de métodos analíticos: Estratégia e Discussão. Pesticidas: R. Ecotoxicol. e Meio Ambiente, Curitiba, v.13, p.129-146, 2003. DOI: http://dx.doi.org/10.5380/pes.13i0.3173

BUDZINSKI, H.; MAZÉAS, O.; TRONCZYNSKI, J.; DÉSAUNAY, Y.; BOCQUENÉ, G.; CLAIREAU, X.. Link between exposure of fish (Solea solea) to PAHs and metabolites: Application to the "Erika" oil spill. Aquatic Living Resources, v.17, p.329-334, 2004. DOI: http://dx.doi.org/10.1051/alr:2004040 
CARUSO, M. S. F.; ALABURDA, J.. Hidrocarbonetos Policíclicos Aromáticos - benzo(a)pireno: uma revisão. Revista do Instituto Adolfo Lutz, v.67, n.1, p.1-27, 2008.

CHEN, B.; HE, R.; YUAN, K.; CHEN, E.; LIN, L.; CHEN, X.; SHA, S.; ZHONG, J.. Polycyclic aromatic hydrocarbons (PAHs) enriching antibiotic resistance genes (ARGs) in the soils. Environmental Pollution, v.220, p.1005-10136, 2017. DOI: http://dx.doi.org/10.1016/i.envpol.2016.11.047

CHUNG, S. Y.; YETELLA, R. R.; KIM, J. S.; KWON, K.; KIM, M. C.; MIN, D. B.. Effects of grilling and roasting on the levels of polycyclic aromatic hydrocarbons in beef and pork. Food Chemistry, v.129, 1420-1426, 2011. DOI: http://dx.doi.org/10.1016/j.foodchem.2011.05.092

EFSA. Scientific Opinion of the Panel on Contaminants in the Food Chain on a request from the European Commission on Polycyclic Aromatic Hydrocarbons in Food. The EFSA Journal, v.724, p.1-114, 2008.

EMBRUSCADO, M. E.. Herbs and spices as antioxidants for food preservation. In: SHAHIDI, F.. Handbook of Antioxidants for Food Preservation. Oxford: Wood head Publishing, 2005. p.251-283. DOI: http://dx.doi.org/10.1016/B978-1-78242-089-7.00011-7

EU. European Union. Commission Regulation. N. 835/2011 of 19 August 2011 amending Regulation (EC) No 1881/2006 as regards maximum levels for polycyclic aromatic hydrocarbons in foodstuffs. Bruxelas: Official Journal of the European Union, 2011.

EU. European Union. Commission Regulation. N. 836/2011 of 19 August 2011 amending Regulation (EC) N 333/2007 laying down the methods of sampling and analysis for the official control of the levels lead, cadmium, mercury, inorganic tin, 3-MCPD and benzo(a)pyrene in foodstuffs. Bruxelas: Official Journal of the European Union, 2011.

EU. European Union. Council Directive 88/388/EEC of 22 June 1988 on the approximation of the laws of the Member States relating to flavorings for use in foodstuffs and to source materials for their production. Bruxelas: Official Journal of the European Union, 1988.

FAO. Food and Agriculture Organization. Codex Alimentarius Commission (CX/CF 08/02/09). Joint FAO/WHO food standards programmer. Codex Committee on Contaminants in Foods, Second Session. FAO, 2008.

FASANO, E.; YEBRA-PIMENTEL, I.; MARTINEZZ-CARBALHO, E. SIMAL-GÂANDARA, J.. Profiling distribution and levels of carcinogenic polycyclic aromatic hydrocarbons in traditional smoked plant and animal foods. Food Control., v.59, p.581590, 2016. DOI:

http://doi.org/10.1016/j.foodcont.2015.06.036

GARCIA, L. P.; GONÇALVES, B. L.; PANHO, G.; SCUSSEL, V. M. Hidrocarbonetos policíclicos aromáticos em alimentos: uma revisão. PUBVET, Londrina, v.8, n.19, 2014.

HARTZELL, S. E.; UNGER, M. A.; VADAS, G. G.; YONKOS, L. T. Evaluating porewater polycyclic aromatic hydrocarbon: related toxicity at a contaminated sediment site using a spiked field-sediment approach. Environmental toxicology and chemistry, v.37, n.3, p.893-902, 2018. DOI:

http://doi.org/10.1002/etc. 4023

HWANG, M-J.; KANG, S-J.; KIM, H-S.; LEE, K. W.. Reduction of the polycyclic aromatic hydrocarbon levels in dried red peppers (Capsicum annuum L.) using heat pump-assisted drying. Food Chemistry, v.297, p.124977, 2019. DOI: http://doi.org/10.1016/j.foodchem.2019.124977

IARC. Agência Internacional de Pesquisa em Câncer. Monographs on the evaluation of the carcinogenic risk of chemicals to humans, overall evaluation of carcinogenicity. Chemical Agents and Related Occupations. Lyon: IARC, 2012.

INCA. Instituto Nacional do Câncer. Hidrocarboneto Policíclico Aromático. Causas e Prevenção. INCA, 2018.

INMETRO. Instituto Nacional de Metrologia, Normalização e Qualidade Industrial. Orientações sobre Validação de Métodos de Ensaios Químicos: DOQ-CGCRE-008. Brasília: INMETRO, 2003.

JAHURUL, M. H. A.; JINAP, S.; ZAIDUL, I. S. M.; SAHENA, F.; FARHADIAN, A.; HAJEB, P.. Determination of fluoranthene, benzo[b]fluoranthene and benzo[a]pyrene in meat and fish products and their intake by Malaysian. Food Bioscience, v.1, p.73-80, 2013. DOI: http://doi.org/10.106/j.fbio.2013.03.006

KUHN, A. V.; COZER, N.; PONT, G. D.; SANDAUSKASHENRIQUE, H.. Gerenciamento de Risco Alimentar e Conscientização Relacionado ao Consumo de Hidrocarbonetos Policíclicos Aromáticos (HPAs) em Pescado. In: ENCONTRO NACIONAL DE PÓS -GRADUAÇÃO - ENPG, 9. Anais. 2020. p.351.

LIN, D.; TU, Y.; ZHU, L.. Concentrations and health risk of polycyclic aromatic hydrocarbons in tea. Journal of Agricultural and Food Chemistry, v.43, p.41-48, 2005. DOI: http://doi.org/10.1016/i.fct.2004.08.010

LONDOÑO, V. A. G.; REYNOSO, C. M.; RESNIK, S. L.. Polycyclic aromatic hydrocarbons (PAHs) survey on tea (Camellia sinensis) commercialized in Argentina. Food Control, v.50, p.31-37, 2015. DOI: http://doi.org/10.1016/i.foodcont.2014.07.036

MARTENA, M. J.; GUTTERS, M. M. P.; GROOT, H. N.; KONINGS, E. J. M.; RIETJENS, I. M. C. M.. Monitoring of Polycyclic Aromatic Hydrocarbons (PAH) in Food Supplements Containing Botanicals and Other Ingredients on the Dutch Market. Food Additives \& Contaminants: Part A, v.28, p.925-942, 2011. DOI: http://dx.doi.org/101080/19440049.2011.569573

MONAGO-MARAÑA, O. PÉREZ, R. L.; ESCANDAR, G. M. PEÑA, A. M. L.; DIAZ, T. G.. Combination of Liquid Chromatography with Multivariate Curve Resolution Alternating Least -Squares (MCR-ALS) in the Quantitation of Polycyclic Aromatic Hydrocarbons Present in Parika Samples. Journal of Agricultural and Food Chemistry, v. 64, n.43, p.8254-8262, 2016. DOI: http://doi.org/10.1021/acs.jafc.6b03852

MORET, S.; PURCARO, G.; CONTE, L. S.. Polycyclic Aromatic hydrocarbons (PAHs) Levels in Propolis and Propolis-based Dietary Supplements from the Italian Market. Food 
Chemistry, v.122, p.333-338, 2010. DOI: http://dx.doi.org/10.1016/i.foodchem.2010.02.041

PEREIRA NETTO, A. D.; MOREIRA, J. C.; DIAS, A. E. X. O.; ARBILLA, G.; FERREIRA, L. F. V.; OLIVEIRA, A. S.; BAREK, J.. Avaliação da contaminação humana por hidrocarbonetos policíclicos aromáticos (HPAs) e seus derivados nitratos (NHPAs): uma revisão metodológica. Química Nova, São Paulo, v.23, n.6, 2000. DOI: http://doi.org/10.1590/S010$\underline{40422000000600010}$

PURCARO, G.; MORE, S.; CONTE, L. S.. Overview on polycyclic aromatic hydrocarbons: Occurrence, legislation and innovative determination in foods. Talanta, v.15, n.105, p.292-305, 2012. DOI:

http://dx.doi/10.1016/j.talanta.2012.10.041

REINHOLDS, I.; PUGAJEVA, I.; BAVRINS, K.; KUCKOVSKA, G.; BARTKEVICS, V.. Mycotoxins, pesticides and toxic metals in commercial spices and herbs. Food Additives \& Contaminants: Part B, v.10, n1, p.5-14, 2016. DOI: http://doi.org/10.1080/19393210.2016.1210244

PIETZSCH, R.. Hidrocarbonetos policíclicos aromáticos em sedimentos recentes da Baía de Guaratuba-PR. 2017.

ROZENTALE, I.; LUN, A. Y.; ZACS, D.; BARTKEVICS, V.. The occurrence of polycyclic aromatic hydrocarbons in dried herbs and spices, Food Control, v.83, p.45-53, 2017. DOI: http://doi.org/10.1016/i.foodcont.2017.04.018

SANCHES FILHO, P. J.; ARDUIM, J.; BETEMPS, G. R.; ANDRADE, G. O.; SILVA, R. C. S.. Evaluation of Polycyclic Aromatic Hydrocarbons in Achyrocline Satureioides (Marcela) and its Infusion. Revista Ibero Americana de Ciências Ambientais, v.12, n.1, p.531-543, 2021. DOI: http://doi.org/10.6008/CBPC2179-6858.2021.001.0043

SCHAARSCHMIDT, S.. Public and private standards for dried culinary herbs and species - Part I: Standards defining the physical and chemical product quality and safety. Food

Control, v.70, p.339-349. DOI:

http://doi.org/10.1016./i.foodcont.2016.06.004

SILVA, S. A.; ROSSI, G. Z.; ALMEIDA, A. P.; GUIZELLINI, G. M.; TORRES, E. A. F. S.; SAMPAIO, G. R.. Benzo(a)Pireno como contaminante em produtos cárneos embutidos comerciais. In: SIMPÓSIO DE SEGURANÇA ALIMENTAR. INOVAÇÃO COM SUSTENTABILIDADE, 7. Anais. 2020. p.27-29.

SUN, R.; SUN, Y.; LI, Q. X.; ZHENG, X.; MAI, B.. Polycyclic aromatic hydrocarbons in sediments and marine organisms: Implications of anthropogenic effects on the coastal environment. Science of the Total Environment, v.640, p.264-272, 2018. DOI: http://dx.doi.org/10.1016/j.scitotenv.2018.05.320

TU, Y. T.; OU, J. H.; TSANG, D. C. W.; DONG, C. D.; CHEN, C. W.; KAO, C. M.. Source identification and ecological impact evaluation of PAHs in urban river sediments: A case study in Taiwan .Chemosphere, v.194, p.666-674, 2018. DOI: http://doi.org/10.1016/i.chemosphere.2017.12.008

UNEP. United Nations Environment Programmer. Determination of petroleum hydrocarbons in sediments. References Methods for Marine Pollution Studies. UNEP, 1992.

USEPA. United States Environmental Protection Agency. Method 8170c- Semivolatile Organic Compounds By Gas Chromatography/Mass Spectrometry (GC/MS). USEPA, 1996.

ZAKI, N.; HAKMAOUI, A.; OUATMANE, A.; FERNANDEZTRUJILIO, J. P.. Quality characteristics of Moroccan sweet paprika (Capsicum annum L.) at different sampling times. Food Science and Technology, Campinas, v.33, n.3, 2013. DOI: http://doi.org/10.1590/S0101-20612013005000072

A CBPC - Companhia Brasileira de Produção Científica (CNPJ: 11.221.422/0001-03) detém os direitos materiais desta publicação. Os direitos referem-se à publicação do trabalho em qualquer parte do mundo, incluindo os direitos às renovaç̃oses, expansões e disseminações da contribuiç̃o, bem como outros direitos subsidiários. Todos os trabalhos publicados eletronicamente poderão posteriormente ser publicados em coletâneas impressas sob coordenação da Sustenere Publishing, da Companhia Brasileira de Produção Científica e seus parceiros autorizados. Os (as) autores (as) preservam os direitos autorais, mas não têm permissão para a publicação da contribuição em outro meio, impresso ou digital, em português ou em tradução. 